\title{
On the deconvolution of the temporal width of laser pulses from pump-probe signals
}

\author{
Henriksen, Niels Engholm; Engel, Volker
}

Published in:

Journal of Chemical Physics

Link to article, DOI:

$10.1063 / 1.480399$

Publication date:

1999

Document Version

Publisher's PDF, also known as Version of record

Link back to DTU Orbit

Citation (APA):

Henriksen, N. E., \& Engel, V. (1999). On the deconvolution of the temporal width of laser pulses from pumpprobe signals. Journal of Chemical Physics, 111(23), 10469-10475. https://doi.org/10.1063/1.480399

\section{General rights}

Copyright and moral rights for the publications made accessible in the public portal are retained by the authors and/or other copyright owners and it is a condition of accessing publications that users recognise and abide by the legal requirements associated with these rights.

- Users may download and print one copy of any publication from the public portal for the purpose of private study or research.

- You may not further distribute the material or use it for any profit-making activity or commercial gain

- You may freely distribute the URL identifying the publication in the public portal

If you believe that this document breaches copyright please contact us providing details, and we will remove access to the work immediately and investigate your claim. 


\title{
On the deconvolution of the temporal width of laser pulses from pump-probe signals
}

\author{
Niels E. Henriksen and Volker Engel ${ }^{\mathrm{a})}$ \\ Department of Chemistry, Technical University of Denmark, DTU 207, DK-2800 Lyngby, Denmark
}

(Received 9 August 1999; accepted 21 September 1999)

\begin{abstract}
We consider pump-probe frequency integrated fluorescence signals. These transients, in general, arise from wave packets where the intrinsic dynamics and the response to the pump pulse is entangled in a complicated way. The signal from an infinitely short $\delta$-pump pulse is the only exception. However, if the pump-probe signal is integrated over the frequencies of the pump laser, the signal can be expressed as a convolution of the pulse envelope with the signal obtained from a $\delta$-pulse excitation. We consider the deconvolution of the pulse envelope and demonstrate that the signal corresponding to a $\delta$-pump pulse can be recovered exactly from signals using pump pulses of finite temporal width. Numerical examples are presented for asymptotic fragment detection in the reaction, ICN $\rightarrow \mathrm{I}+\mathrm{CN}$. (C) 1999 American Institute of Physics. [S0021-9606(99)00747-3]
\end{abstract}

\section{INTRODUCTION}

The dynamics of elementary physical and chemical processes can be followed in real time by femtosecond pumpprobe spectroscopy. ${ }^{1-5}$ From these measurements one can extract various characteristic times, e.g., the time required to break a chemical bond. In an ideal measurement, one should be able to launch a wave packet on a repulsive potential at a well-defined time and, subsequently, record the time it takes for the center of this wave packet to move to a position corresponding to a certain internuclear distance. The dissociation time will, obviously, depend on the chosen repulsive potential as well as on the distance chosen as the one defining bond-breaking. The signal obtained for an instantaneous excitation in time, that is, a $\delta$-pump pulse, is required in order to establish the rigorous definition of the zero of time in pump-probe spectroscopy. A $\delta$-pump pulse creates a Franck-Condon wave packet, that is a replica of the initial state times the transition dipole moment to an electronically excited state, where this wave packet subsequently evolves in time.

The ideal $\delta$-pulse is not available in practice, therefore it is important to understand how, for example, a pump pulse with a finite temporal width affects the pump-probe signal. It has already been demonstrated that the pump-probe signal can depend quite strongly on the parameters of the pump laser.

It is often assumed that the combined effect of two contributions to an experimental signal can be expressed as a convolution of the individual contributions. ${ }^{7}$ Specifically, in the field of pump-probe spectroscopy, it has been suggested ${ }^{8}$ that for a pump pulse with finite width, the pump-probe signal can be analyzed in the following way: The signal has a certain time-dependence $S_{\mathrm{FC}}(t)$ for an infinitely narrow $\delta$-pump pulse. When the pump pulse has a finite width, its intensity has a time-dependence given by $I(t)$, and now each

\footnotetext{
a)Permanent address: Institut für Physikalische Chemie, Universität Würzburg, Am Hubland, D-97074 Würzburg, Germany.
}

molecule is transferred to the excited state at a slightly different time, $t^{\prime}$. The resulting signal at time $t$ is a sum of terms of the form $I\left(t^{\prime}\right) S_{\mathrm{FC}}\left(t-t^{\prime}\right)$. This sum (integral) takes the form of a convolution of the intensity $I(t)$ with the $\delta$-pulse signal $S_{\mathrm{FC}}(t)$. The main purpose of the present article, is to consider the validity of such an approach within the framework of quantum mechanics. In order to enhance insight in the limit of an ultrashort pump pulse, we derive an analytical expression for the pump-probe frequency integrated fluorescence signal for asymptotic fragment detection. This expression illustrates that for a fixed carrier frequency of the pump pulse, the signals arise from wave packets which are based on a complicated interplay between the intrinsic dynamics and the response to the pump pulse. The signal $S_{\mathrm{FC}}(t)$ for a $\delta$-pump pulse is the only exception.

This article is organized in the following way: Sec. II contains, for an ultrashort pump pulse, the derivation of an analytical expression for the pump-probe signal for asymptotic fragment detection. In Sec. III, we leave the analytical model and demonstrate that the pump-probe signal can be expressed as a convolution of the pulse envelope with the signal corresponding to a $\delta$-pump pulse, if the signal for finite pump pulses is integrated over all pump frequencies. Section IV presents numerical examples illustrating that an exact deconvolution of the pump pulse is possible. Finally, Sec. V contains a summary and a conclusion.

\section{PUMP-PROBE SIGNALS}

\section{A. General expressions}

We consider, as illustrated in Fig. 1, the interaction between a molecule and two time-delayed pulses - a pump and a probe pulse. Within the electric dipole approximation, the field-molecule coupling terms take the form (for absorption)

$$
\begin{aligned}
& C_{\text {pump }}(t)=-(1 / 2) \mu_{10} a(t) e^{-i \omega_{1} t}, \\
& C_{\text {probe }}(t)=-(1 / 2) \mu_{21} a(t-T) e^{-i \omega_{2}(t-T)},
\end{aligned}
$$




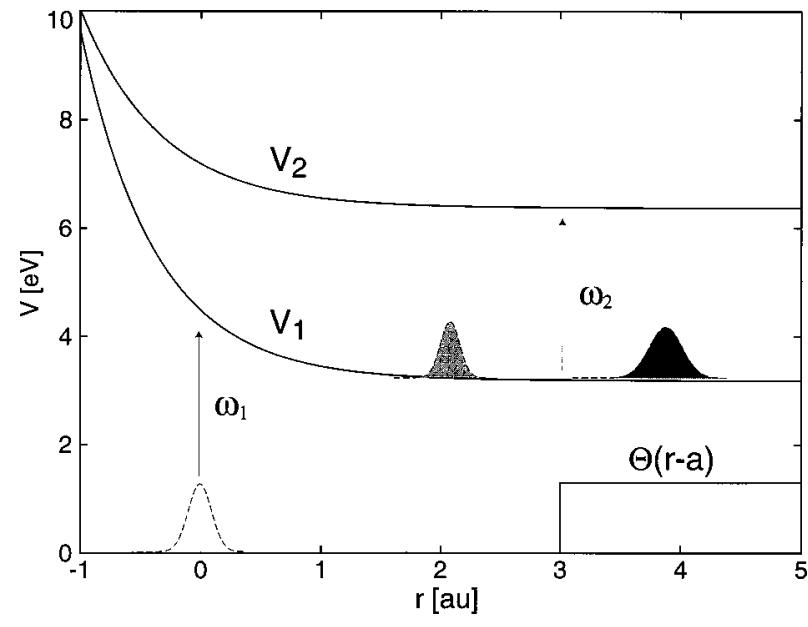

FIG. 1. Pump-probe scheme for direct dissociation: the pump pulse $\left(\omega_{1}\right)$ prepares a wave packet in an excited electronic state with potential curve $V_{1}$. The probe pulse $\left(\omega_{2}\right)$ induces a transition to another electronic state $\left(V_{2}\right)$ and the total fluorescence from this state is measured. The probe-laser frequency is here chosen such that the wave packet is probed when it enters the asymptotic region where the distance $r$ exceeds a critical point $a$. This spatial window is denoted as $\Theta(r-a)$. The potentials represent a simple model for ICN dissociation where $r$ is the $\mathrm{I}-\mathrm{C}$ distance relative to the equilibrium position in the electronic ground state.

where $\omega_{1}$ and $\omega_{2}$ are the carrier frequencies, $T$ is the delay time between the pulses, $\mu_{10}$ and $\mu_{21}$ are the projections of the transition dipole moments on the polarization of the electric field vector, and $a(t)$ is the pulse envelope.

The pump pulse creates a wave packet which, according to first-order perturbation theory, can be written in the form

$$
\begin{aligned}
\left|\psi_{1}(t)\right\rangle= & \frac{i}{2 \hbar} \int_{0}^{t} d t^{\prime} e^{-i\left(\hbar \omega_{1}+\epsilon_{0}\right) t^{\prime} / \hbar} a\left(t^{\prime}\right) \\
& \times \exp \left(-i \hat{H}_{1}\left(t-t^{\prime}\right) / \hbar\right)\left|\phi_{0}(0)\right\rangle,
\end{aligned}
$$

where

$$
\left|\phi_{0}(0)\right\rangle=\mu_{10}\left|\psi_{0}(0)\right\rangle \text {. }
$$

$\left|\psi_{0}(0)\right\rangle$ is the initial stationary vibrational/rotational eigenstate of the molecule, $\epsilon_{0}$ is the energy of this state, and $\hat{H}_{1}$ is the Hamiltonian for nuclear motion in electronic state (1). Equation (2) shows that the wave packet created by the pump pulse can be viewed as a superposition of FranckCondon wave packets created over a period of time defined by the pulse envelope. It is a coherent superposition, however, which means that interference terms between FranckCondon wave packets created at different times show up in the probability amplitude.

The probe pulse creates a new nonstationary state $\left|\psi_{2}(t)\right\rangle$. In the limit of nonoverlapping pump and probe pulses which we will consider in the present article, this state can be calculated according to first-order perturbation theory, now with $\left|\psi_{1}(t)\right\rangle$ as initial state. The total pump-probe fluorescence signal $P(T)$ is assumed to be proportional to the norm of $\left|\psi_{2}(t)\right\rangle$ after the probe pulse has decayed to zero,

$$
P(T)=\lim _{t \rightarrow \infty} \int d r\left|\psi_{2}(r, t)\right|^{2} .
$$

If one neglects commutators between the kinetic energy operators and the $r$-dependent terms in the perturbation integral (pump-probe signal in the limit of short probe pulse), the signal can be written as ${ }^{9-13}$

$$
P(T)=\int d r\left|F\left(D(r)-\omega_{2}\right)\right|^{2}\left|\phi_{1}(r, T)\right|^{2},
$$

where $\left|\phi_{1}(T)\right\rangle=\mu_{21}\left|\psi_{1}(T)\right\rangle$,

and

$$
F\left(D(r)-\omega_{2}\right)=\int_{-\infty}^{+\infty} d t a(t) e^{i\left(D(r)-\hbar \omega_{2}\right) t / \hbar} .
$$

Here $D(r)=V_{2}(r)-V_{1}(r)$ is the difference between the potential energy curves in the electronic states (2) and (1). Thus, the signal measures the norm of the wave packet $\phi_{1}$ at time $T$ within the window $F\left(D(r)-\omega_{2}\right)$ (Franck-Condon window). In the case of fragment detection for a dissociation process, the carrier frequency of the probe laser matches the asymptotic value of the difference potential.

\section{B. An ultrashort pump pulse}

In this section, we will show that in the limit of an ultrashort pump pulse the general form of the pump-probe signal can be obtained analytically.

We write Eq. (2) in the form

$$
\begin{aligned}
\left|\psi_{1}(t)\right\rangle= & \frac{i}{2 \hbar} \exp \left(-i \hat{H}_{1} t / \hbar\right) \int_{0}^{t} d t^{\prime} e^{-i\left(\hbar \omega_{1}+\epsilon_{0}\right) t^{\prime} / \hbar} \\
& \times a\left(t^{\prime}\right) \exp \left(i \hat{H}_{1} t^{\prime} / \hbar\right)\left|\phi_{0}(0)\right\rangle .
\end{aligned}
$$

The pulse envelope centered at the time $t=t_{p}$, is represented by a Gaussian form,

$$
a(t)=E_{0} \sqrt{(\gamma / \pi)} \exp \left[-\gamma\left(t-t_{p}\right)^{2}\right],
$$

where $\gamma$ is large, corresponding to an ultrashort pulse. A one-dimensional model is considered and for a molecule in the vibrational ground state, $\phi_{0}(r, 0)$ is approximately a Gaussian, that is

$$
\phi_{0}(r, 0)=\exp \left\{(i / \hbar)\left[A_{0}\left(r-r_{0}\right)^{2}+s_{0}\right]\right\},
$$

where $A_{0}=i \operatorname{Im}\left(A_{0}\right)=i m \omega / 2, \omega$ is the vibrational frequency, $m$ is the mass, and normalization implies that $s_{0}$ $=(i \hbar / 4) \ln \left[\pi \hbar /\left(2 \operatorname{Im}\left(A_{0}\right)\right)\right]$.

We assume now that the dynamics can be described within a (time-dependent) local harmonic approximation to the potential. Thus, $\left\langle r\left|\exp \left(i \hat{H}_{1} t^{\prime} / \hbar\right)\right| \phi_{0}(0)\right\rangle$, which corresponds to propagation backwards in time, can be represented by the complex conjugate of a Gaussian wave packet of the form $\left\langle r \mid G\left(t^{\prime}\right)\right\rangle^{*}=\left\langle r \mid G\left(-t^{\prime}\right)\right\rangle$, where ${ }^{14,15}$

$$
\begin{aligned}
\left\langle r \mid G\left(-t^{\prime}\right)\right\rangle= & \exp \left\{( - i / \hbar ) \left[A_{t^{\prime}}^{*}\left(r-r_{t^{\prime}}\right)^{2}\right.\right. \\
& \left.\left.+p_{t^{\prime}}\left(r-r_{t^{\prime}}\right)+s_{t^{\prime}}^{*}\right]\right\} .
\end{aligned}
$$

The time-evolution of the parameters is given by simple equations of motion. For example, the center of the wave packet $\left(r_{t}, p_{t}\right)$ evolves in time according to Hamilton's equations. The change in a short time step $\tau$, starting at $t=0$ is 
obtained by setting $t=0$ in the right-hand side of these equations of motion. We get (neglecting the spreading of the wave packet $)^{16}$

$$
\begin{aligned}
& r_{\tau}=r_{0}, \\
& p_{\tau}=-\beta \tau, \\
& A_{\tau}=A_{0}, \\
& s_{\tau}=s_{0}-\left(V_{1}\left(r_{0}\right)+\hbar \omega / 2\right) \tau,
\end{aligned}
$$

where $\beta=\partial V_{1}(r) /\left.\partial r\right|_{r=r_{0}}$ is the derivative of the potential $V_{1}(r)$ at $r=r_{0}$. For $t^{\prime}$ close to zero, the Gaussian wave packet takes the form

$$
\begin{aligned}
\left\langle r \mid G\left(-t^{\prime}\right)\right\rangle= & \exp \left\{( - i / \hbar ) \left[A_{0}^{*}\left(r-r_{0}\right)^{2}\right.\right. \\
& \left.\left.-\beta t^{\prime}\left(r-r_{0}\right)+s_{0}^{*}-V_{1}^{\prime}\left(r_{0}\right) t^{\prime}\right]\right\},
\end{aligned}
$$

where $V_{1}^{\prime}\left(r_{0}\right)=V_{1}\left(r_{0}\right)+\hbar \omega / 2$. The pulse is assumed to be so short that we can use this approximation in the evaluation of the integral in Eq. (7). In practice, the short-time approximation above often suffices for the description of the total width of an absorption spectrum, that is the initial decay of the autocorrelation function. ${ }^{17}$ Thus, "an ultrashort pulse" in the present context means a pulse so short that the pulse envelope has raised and decayed to zero before the initial decay of the autocorrelation function. This is fulfilled in the limit $\gamma$ large and $t_{p} \rightarrow 0$.

After evaluation of the integral in Eq. (7), the result can be written in the form,

$$
\left|\psi_{1}(t)\right\rangle=\frac{i E_{0}}{2 \hbar} \exp \left(-i \hat{H}_{1} t / \hbar\right)\left|\Phi_{0}(0)\right\rangle,
$$

where

$$
\begin{aligned}
\left\langle r \mid \Phi_{0}(0)\right\rangle= & \exp \left\{-m \omega\left(r-r_{0}\right)^{2} /(2 \hbar)+i s_{0} / \hbar\right\} \\
& \times \exp \left\{-\beta^{2}\left(r-r_{0}-r_{e}\right)^{2} /\left(4 \hbar^{2} \gamma\right)\right. \\
& \left.+i t_{p} \beta\left(r-r_{0}-r_{e}\right) / \hbar\right\},
\end{aligned}
$$

and $r_{e}=\left[\hbar \omega_{1}-V_{1}\left(r_{0}\right)\right] / \beta$. Thus, the obtained wave function is a product of two Gaussians, the initial Gaussian, i.e., the Gaussian generated by a $\delta$-pump pulse times a displaced Gaussian where the displacement is proportional to the detuning $\left[\hbar \omega_{1}-V_{1}\left(r_{0}\right)\right]$.

For $\hbar \omega_{1}=V_{1}\left(r_{0}\right)$, that is "on-resonant" pumping at the center of the absorption band, $\left\langle r \mid \Phi_{0}(0)\right\rangle$ is again a single Gaussian with the same expectation value of the position as in the initial state in Eq. (9), that is, $r_{0}$. The expectation value of the momentum has, however, changed from 0 to $t_{p} \beta$. The width of the wave packet has also changed from $\hbar /\left[4 \operatorname{Im}\left(A_{0}\right)\right]$ to $(\Delta r)_{0}^{2}=\hbar /\left[4 \operatorname{Im}\left(A_{0}\right)+\beta^{2} /(\hbar \gamma)\right]$, that is the width is reduced compared to the Franck-Condon wave packet. Since the Gaussian is still a minimum uncertainty packet, the reduced width implies that an ultrashort pulse as defined above, produces a squeezed state. Similar squeezing phenomena have been discussed previously based on analytical models ${ }^{18}$ and numerical simulations. ${ }^{19}$ Note that in the limit where $\gamma \rightarrow \infty$ and $t_{p} \rightarrow 0$, corresponding to a $\delta$-pump pulse, Eq. (14) reduces, as expected, to the Franck-Condon wave packet.
We consider now fragment detection for a dissociation process, where the carrier frequency of the probe laser matches the asymptotic value of the difference potential. To a good approximation, the detection window can be replaced by a theta-function. Thus, $F\left(D(r)-\omega_{2}\right)=\theta(r-a)$, so that the pump-probe signal becomes

$$
P(T)=\int_{a}^{\infty} d r\left|\psi_{1}(r, T)\right|^{2},
$$

where the transition dipole moment is set to unity. We consider on-resonant pumping where $r_{e}=0$ in Eq. (14), and assume again that the dynamics can be described within a (time-dependent) local harmonic approximation to the potential. Then $\left\langle r \mid \psi_{1}(t)\right\rangle$ can at all times be represented by the Gaussian form, Eq. (10). The integral in Eq. (15) then takes the form ${ }^{16}$

$$
P(T)=\frac{E_{0}^{2}}{4 \hbar^{2}\left(2 \pi(\Delta r)_{T}^{2}\right)^{1 / 2}} \int_{a}^{\infty} d r \exp \left[-\frac{\left(r-r_{T}\right)^{2}}{2(\Delta r)_{T}^{2}}\right],
$$

which can be expressed as

$$
P(T)=\frac{E_{0}^{2}}{8 \hbar^{2}}\left(1+\operatorname{erf}\left(\frac{r_{T}-a}{\sqrt{2}(\Delta r)_{T}}\right)\right),
$$

where the error function is defined by

$$
\operatorname{erf}(x)=\frac{2}{\sqrt{\pi}} \int_{0}^{x} e^{-u^{2}} d u .
$$

We observe that, at the time when the center of the wave packet has reached the detection window, that is $r_{T}=a$, $P(T)$ is at half of its asymptotic value. It should also be noted that the time-derivative of the signal at this point is given by the ratio between the mean speed and the uncertainty $(\Delta r)_{T} \cdot{ }^{16}$ This ratio gives the intrinsic time-resolution according to quantum mechanics.

This analytical model shows that the initial values of the Gaussian wave packet parameters depend on the form of the pump laser. The expression illustrates, accordingly, that the response to the pump pulse and the intrinsic dynamics is entangled in a complicated way, with the $\delta$-pump pulse as the only exception. Thus, at a given pump frequency, there seems to be no way to "eliminate" the dependence on the pulse width.

\section{PUMP-PROBE SIGNALS INTEGRATED OVER PUMP FREQUENCIES}

\section{A. The convoluted signal}

We will now show that it is possible to extract the signal for a Franck-Condon wave packet from measurements using pump pulses of finite temporal width. We write Eq. (5) in the form,

$$
P\left(\omega_{1}, T\right)=\left\langle\psi_{1}(T)|A(r)| \psi_{1}(T)\right\rangle,
$$

where

$$
A(r)=\left|F\left(D(r)-\omega_{2}\right)\right|^{2}\left[\mu_{21}(r)\right]^{2} .
$$


Now using the first-order expression in Eq. (2) for the wave packet,

$$
\begin{aligned}
P\left(\omega_{1}, T\right)= & \frac{1}{4 \hbar^{2}} \int_{0}^{T} d t^{\prime \prime} \int_{0}^{T} d t^{\prime} e^{i \omega_{1}\left(t^{\prime \prime}-t^{\prime}\right)} a\left(t^{\prime}\right) a\left(t^{\prime \prime}\right) \\
& \times\left\langle M\left(T-t^{\prime \prime}\right)|A(r)| M\left(T-t^{\prime}\right)\right\rangle,
\end{aligned}
$$

where

$$
|M(T-t)\rangle=\exp \left(-i \hat{H}_{1}(T-t) / \hbar\right)\left|\phi_{0}(0)\right\rangle,
$$

is the Franck-Condon wave packet associated with a $\delta$-pump pulse excitation. This expression shows that, at a given pump frequency, quantum mechanical interference terms show up between Franck-Condon wave packets created at different times. Furthermore, these "off-diagonal", terms are frequency dependent.

We integrate now over the frequencies of the pump laser. The signal $S(T)$ can then be written as

$$
\begin{aligned}
S(T)= & \int_{-\infty}^{\infty} P\left(\omega_{1}, T\right) d \omega_{1} \\
= & \frac{\pi}{2 \hbar^{2}} \int_{0}^{T} d t^{\prime \prime} \int_{0}^{T} d t^{\prime} \delta\left(t^{\prime \prime}-t^{\prime}\right) a\left(t^{\prime}\right) a\left(t^{\prime \prime}\right) \\
& \times\left\langle M\left(T-t^{\prime \prime}\right)|A(r)| M\left(T-t^{\prime}\right)\right\rangle \\
= & \frac{\pi}{2 \hbar^{2}} \int_{0}^{T} d t^{\prime}\left[a\left(t^{\prime}\right)\right]^{2}\left\langle M\left(T-t^{\prime}\right)|A(r)| M\left(T-t^{\prime}\right)\right\rangle .
\end{aligned}
$$

This is an integral of signals for Franck-Condon wave packets created at different times with amplitudes given by the square of the pulse envelope. Note that $P\left(\omega_{1}, T\right)$ is only nonzero within the absorption band of electronic state (1), therefore the integration over frequency can be extended to infinity.

This expression can be rewritten as a convolution,

$$
\begin{aligned}
S(T) & =\frac{\pi}{2 \hbar^{2}} \int_{-\infty}^{T} d t^{\prime}\left[a\left(t^{\prime}\right)\right]^{2}\left\langle M\left(T-t^{\prime}\right)|A(r)| M\left(T-t^{\prime}\right)\right\rangle \\
& =\frac{\pi}{2 \hbar^{2}} \int_{0}^{\infty} d y[a(T-y)]^{2}\langle M(y)|A(r)| M(y)\rangle \\
& =\frac{\pi}{2 \hbar^{2}} \int_{-\infty}^{\infty} d y[a(T-y)]^{2} \Theta(y)\langle M(y)|A(r)| M(y)\rangle,
\end{aligned}
$$

where we used that $a(t)=0$ for $t<0$, changed variable to $y=T-t^{\prime}$ in the second line, and introduced a theta-function in the last line, since the dynamics induced by the pump pulse is absent for $y<0$. Thus, the frequency integrated signal is expressed as a convolution between the square of the pulse envelope and the signal for a Franck-Condon wave packet,

$$
S_{\mathrm{FC}}(t)=\Theta(t)\langle M(t)|A(r)| M(t)\rangle .
$$

In order to obtain the frequency integrated signal experimentally, it is necessary to make a series of measurements covering the entire absorption band.

\section{B. Deconvolution}

The deconvolution of the pump pulse is, in principle, straightforward using some basic rules of the Fourier transformation. ${ }^{7}$ Thus, the Fourier transform of a convolution is equal to the product of the Fourier transforms of each function. This product can then simply be divided by the Fourier transform of the squared pulse envelope, in order to isolate the desired signal for the Franck-Condon wave packet.

For asymptotic fragment detection, the signal $S(T)$ will approach a constant value for $T \rightarrow \infty$. In this case, it is more convenient to consider the time-derivative of the original signal,

$$
\frac{d S(T)}{d T}=\frac{d}{d T} \int_{-\infty}^{\infty} P\left(\omega_{1}, T\right) d \omega_{1} .
$$

This function decays to zero when the entire wave packet has entered the detection window. Thus, it is straightforward to evaluate its Fourier transform $f(\omega)$,

$$
\begin{aligned}
f(\omega) & =\int_{-\infty}^{\infty}[d S(T) / d T] e^{-i \omega T} d T \\
& =i \omega \widetilde{S}(\omega)+S\left(T_{f}\right) e^{-i \omega T_{f}},
\end{aligned}
$$

$T_{f}$ is the final time considered, where $S(T)$ has reached its constant value, and "tilde" denotes the Fourier transform. Using Eq. (24), we get

$$
\widetilde{S}(\omega)=\tilde{a}(\omega) \widetilde{S}_{\mathrm{FC}}(\omega)=f(\omega) /(i \omega)-S\left(T_{f}\right) e^{-i \omega T_{f}}(i \omega),
$$

where $\tilde{a}(\omega)$ and $\widetilde{S}_{\mathrm{FC}}(\omega)$ are the Fourier transforms of $a(t)^{2}$ and $S_{\mathrm{FC}}(t)$, respectively. If we, at this stage, apply an inverse Fourier transform to each term in order to get back to $S(T)$, we notice that the Fourier transform of the last term can be evaluated analytically using the integral: $\int_{0}^{\infty} d x \sin [a x] / x$ $=-\pi / 2$, for $a<0$. Thus, the last term corresponds merely to the addition of the constant $S\left(T_{f}\right) / 2$ in the time domain. This term can, consequently, be omitted in the following.

If the square of the pulse envelope is a Gaussian centered at the time $t=t_{p}$,

$$
a(t)^{2}=E_{0}^{2} \sqrt{(\alpha / \pi)} \exp \left[-\alpha\left(t-t_{p}\right)^{2}\right],
$$

the Fourier transform of this function becomes

$$
\tilde{a}(\omega)=\int_{-\infty}^{\infty} a(t)^{2} e^{-i \omega t} d t=E_{0}^{2} \exp \left[-\omega^{2} /(4 \alpha)\right] e^{-i \omega t} p,
$$

and one can now divide both sides in Eq. (28) by $\widetilde{a}(\omega)$ and, finally, make an inverse Fourier transform of $\widetilde{S}_{\mathrm{FC}}(\omega)$ in order to get $S_{\mathrm{FC}}(T)$.

We consider the numerical implementation in the next section. It should be noted that experimental data can be contaminated with various forms of noise. Thus, the deconvolution of experimental data will, in general, be more cum- 
bersome numerically, than illustrated in the next section where theoretical data, free from noise, is used as input for the deconvolution.

\section{NUMERICAL EXAMPLES}

The dissociation of ICN into $\mathrm{I}+\mathrm{CN}$ is chosen as a numerical example. In the first gas phase femtosecond timeresolved experiments, Zewail and co-workers investigated this system. ${ }^{20,21}$ Quantum calculations were performed within a one-dimensional model by Williams and Imre ${ }^{22}$ and a good overall agreement was found with the data. Later it was shown by Heather and Metiu ${ }^{23}$ that the rotational degrees of freedom have to be considered to explain finer details of the experiment. Here we use the simple onedimensional model, and the employed potential curves are displayed in Fig. 1, as a function of the I-C separation using the equilibrium distance in the electronic ground state as the origin of the axis. The origin of the energy scale corresponds to the energy of the initial wave function $\psi_{0}$ in the electronic ground state. The initial state was taken to be a Gaussian centered at $r=0$ with a width (full width at half maximum) of 0.15 a.u. We solve the time-dependent Schrödinger equation with the split-operator method ${ }^{24}$ and the time-integrals which appear in perturbation theory are calculated as described in Ref. 25. The transition dipole moments were set to unity in all calculations and a Gaussian envelope function for the pump pulse was employed throughout. The detection window starts at $a=3$ a.u.

Figure 2(a) shows the almost exact agreement between the pump-probe signal for a $\delta$-pump pulse (solid line) obtained by numerical solution of the Schrödinger equation, and the result obtained from the analytical expression in Eq. (17). As pointed out above, the slope of the signal depends on the width of the wave packet and, consequently, the spreading of the wave packet as it travels from the initial position into the detection window. The excellent agreement shows that the time-dependent local harmonic approximation to the exponential potential is well fulfilled in the present case.

Figure 2(b) shows the pump-probe signal for a $\delta$-pump pulse (solid line) and the result obtained by deconvolution from a frequency integrated signal [Eq. (23)] calculated with a $30 \mathrm{fs}$ (full width at half maximum) Gaussian pump pulse centered at $t_{p}=60 \mathrm{fs}$. The agreement is perfect and within the numerical resolution of the calculation. The small oscillations in the deconvoluted signal are due to numerical errors. The absolute value of the Fourier transform in Eq. (27) decays to zero for large frequencies. In order to deconvolute, these small values which can be beyond the numerical accuracy of the Fourier transform, must be divided by the Fourier transform of the pulse given by Eq. (30). A simple way to circumvent this problem is to set the values of the Fourier transform to zero, when the absolute value is small and beyond the numerical accuracy. The oscillations could have been removed or reduced by the use of an appropriate window function. It should be noted that, essentially, the same good agreement is obtained from a deconvolution, not from the frequency integrated signal but from a signal corresponding to a pump frequency at the maximum of the absorption

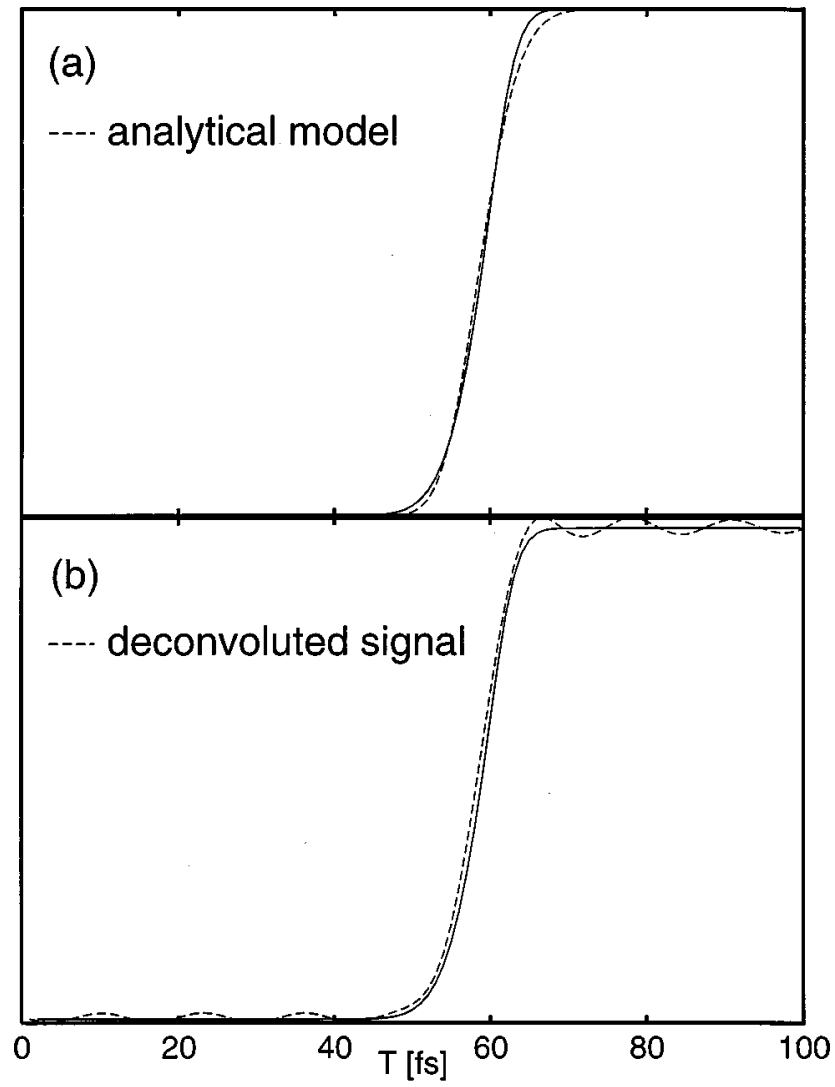

FIG. 2. Panel (a) shows the result of the analytical model compared to the numerically calculated pump-probe signal for a $\delta$-pump pulse (solid line). Panel (b) contains the pump-probe signal deconvoluted from a signal obtained with a $30 \mathrm{fs}$ (full width at half maximum) Gaussian pump pulse centered at $t_{p}=60 \mathrm{fs}$. The pump-probe signal for a $\delta$-pump pulse is also shown (solid line).

spectrum. The absorption band for ICN is fairly narrow and symmetric and, in fact, a deconvolution at any pump frequency within this band will give a result which is fairly close to the signal given in Fig. 2.

Figure 3 illustrates, however, that the pump-probe signal can depend quite strongly on the frequency of the pump laser. In this calculation the width of the vibrational ground state has been set to 0.5 a.u. Pump-probe signals are shown for a Gaussian pump pulse centered at $t_{p}=60 \mathrm{fs}$ with a duration of $30 \mathrm{fs}$ (full width at half maximum). The center frequencies of the pump laser are specified in the figure. In the insert in the upper left corner is shown the pump-probe signals obtained from a deconvolution of the convoluted signals at the three pump frequencies. The pump frequencies are at the maximum $(4.4 \mathrm{eV})$ and the wings of the absorption band $(4.0$ and $5.1 \mathrm{eV})$. The dissociation time inferred from the three deconvoluted signals vary by about $30 \%$. It should be noted that the deconvoluted signal obtained from the pump frequency at the maximum of the absorption band is quite close to the signal for a $\delta$-pump pulse. This is again due to the symmetric shape of the absorption band. However, in general, one will only get the desired signal for a $\delta$-pump pulse by deconvolution from the frequency integrated signal.

Finally, in order to establish a dissociation time it might be tempting to use a simpler "deconvolution" of the finite temporal width of the pump pulse. Figure 4 shows pump- 


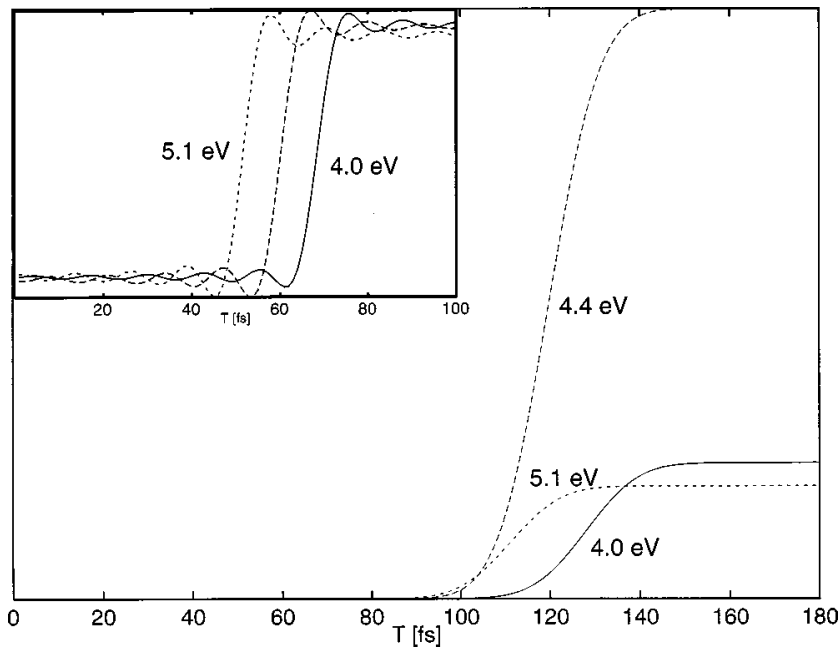

FIG. 3. An illustration of the frequency dependence of the pump-probe signal. In this calculation the width of the vibrational ground state has been set to 0.5 a.u. Pump-probe signals are shown for a Gaussian pump pulse centered at $t_{p}=60 \mathrm{fs}$ with a duration of $30 \mathrm{fs}$ (full width at half maximum). The center frequencies of the pump laser are specified in the figure. The insert in the upper left corner shows the normalized pump-probe signals for $\delta$-pulse excitation obtained from a deconvolution of the convoluted signals at the three pump frequencies.

probe signals for a $\delta$-pump pulse (solid line) and for 30 and 60 fs Gaussian pump pulses (full width at half maximum). The width of the vibrational ground state is again 0.15 a.u. In the plot, the zero of time is chosen such that it is at the maximum of the envelope function for the respective pump pulses. The broadening of the signal due to finite pulse widths is clearly seen in the figure. In addition, it is observed that the three curves cross almost at the same point close to a time of $60 \mathrm{fs}$, that is, close to the dissociation time which could be inferred from the exact deconvolution. It should, however, be noted that a symmetric Gaussian pump pulse was used and a similar agreement would not have been obtained with a nonsymmetric pump pulse. Furthermore, the frequency of the 30 and $60 \mathrm{fs}$ pump pulses was chosen to be at the maximum of the absorption band $(4.5 \mathrm{eV})$, and one should keep in mind that, in general, the signals will be frequency dependent, as illustrated in Fig. 3

\section{CONCLUSIONS}

The main result of this work is that the total (integrated) pump-probe fluorescence signal can be expressed as a convolution between the square of the pulse envelope and the signal for a Franck-Condon wave packet. This is an exact statement when the signal is integrated over the frequency of the pump laser, that is, over the entire absorption band. This result was derived in the limit of nonoverlapping pump and probe pulses, and in the limit of a short probe pulse. When the signal is expressed as a convolution it is possible to eliminate the "blurring" due to the finite duration of the pump pulse. The present work has, accordingly, established an exact procedure for the extraction of the signal for a Franck-Condon wave packet from measurements using a pump pulse of finite temporal width.

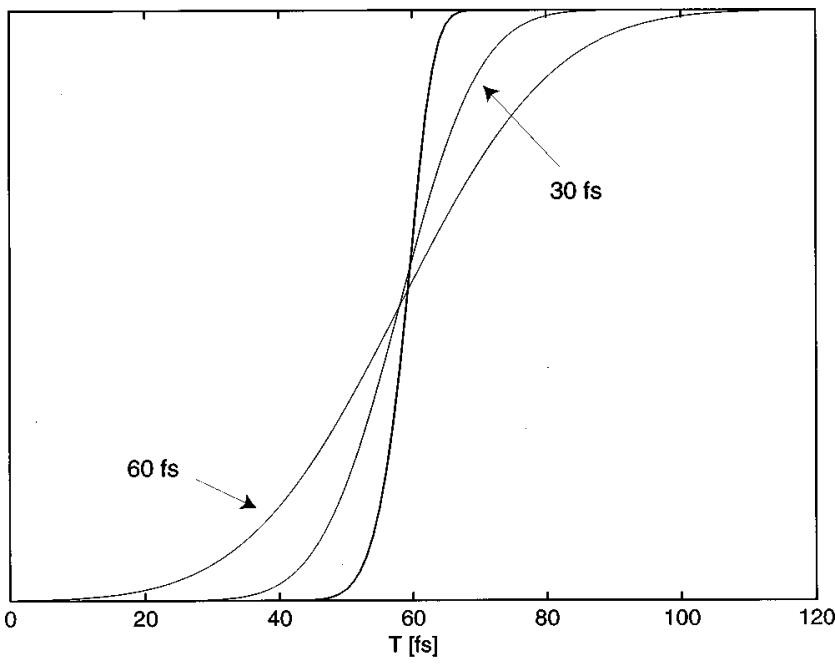

FIG. 4. Pump-probe signal for a $\delta$-pump pulse (solid line) and for 30 and $60 \mathrm{fs}$ Gaussian pump pulses (full width at half maximum). All signals were normalized to the same asymptotic value. The zero of time is chosen such that it is at the peak of the pump pulse.

A similar deconvolution has been suggested previously ${ }^{8}$ using a classical picture of the excitation due to the pump pulse, that is, quantum mechanical interferences were neglected. As illustrated by the numerical examples for asymptotic fragment detection in $\mathrm{ICN} \rightarrow \mathrm{I}+\mathrm{CN}$, this approach happens to work quite well for this system, especially for pump frequencies around the maximum of the absorption band. However, in general, one will only get the desired signal associated with a Franck-Condon wave packet from a deconvolution where a signal integrated over the whole absorption band is used as input.

We considered a one-dimensional model of ICN in the numerical examples. It is perhaps worthwhile to stress that the deconvolution approach presented in Sec. III can be applied to multidimensional systems just as easily as in the examples presented in this article.

Finally, we derived an analytical expression for the pump-probe signal in the case of asymptotic fragment detection. An ultrashort pump pulse in a regime close to the $\delta$-pump pulse was considered. The derivation was based on a time-dependent local harmonic approximation to the potential. The wave packet created by the ultrashort pump pulse has the same average position as the initial state but it is squeezed in position space, except in the extreme limit of a $\delta$-pump pulse. We applied this analytical model to fragment detection in $\mathrm{ICN} \rightarrow \mathrm{I}+\mathrm{CN}$, and found excellent agreement with the numerically calculated pump-probe signal for a $\delta$-pump pulse.

\section{ACKNOWLEDGMENTS}

Financial support by the Deutsche Forschungsgemeinschaft and the Fonds der Chemischen Industrie is gratefully acknowledged. V. E. thanks the Technical University of Denmark at Lyngby for the opportunity to participate in its program for visiting professors and for the hospitality during his visit at the Department of Chemistry. 
${ }^{1}$ A. H. Zewail, Femtochemistry (World Scientific, Singapore, 1994), Vol. $1,2$.

${ }^{2}$ Femtosecond Chemistry, edited by J. Manz and L. Wöste (VCH, Weinheim, 1995).

${ }^{3}$ Femtochemistry, edited by M. Chergui (World Scientific, Singapore, 1996).

${ }^{4}$ Femtochemistry and Femtobiology, edited by V. Sundström (Imperial College Press, London, 1997).

${ }^{5}$ Chemical Reactions and their Control on the Femtosecond Time Scale, XXth Solvay Conference on Chemistry, edited by P. Gaspard and I. Burghardt [Adv. Chem. Phys. 101, 3 (1997)].

${ }^{6}$ J. L. Krause, M. Shapiro, and R. Bersohn, J. Chem. Phys. 94, 5499 (1991).

${ }^{7}$ D. C. Champeney, Fourier Transforms and their Physical Applications (Academic, London, 1973).

${ }^{8}$ M. J. Rosker, M. Dantus, and A. H. Zewail, J. Chem. Phys. 89, 6113 (1988).

${ }^{9}$ M. Braun, C. Meier, and V. Engel, J. Chem. Phys. 103, 7907 (1995).

${ }^{10}$ Z. Li, J.-Y. Fang, and C. C. Martens, J. Chem. Phys. 104, 6919 (1996).
${ }^{11}$ E. M. Hiller and J. A. Cina, J. Chem. Phys. 105, 3419 (1996).

${ }^{12}$ J. Cao and K. R. Wilson, J. Chem. Phys. 106, 5062 (1997).

${ }^{13}$ H. Dietz and V. Engel, J. Phys. Chem. A 102, 7406 (1998).

${ }^{14}$ E. J. Heller, J. Chem. Phys. 62, 1544 (1975).

${ }^{15}$ K. B. Møller and N. E. Henriksen, J. Chem. Phys. 105, 5037 (1996).

${ }^{16}$ N. E. Henriksen, Adv. Chem. Phys. 91, 433 (1995).

${ }^{17} \mathrm{R}$. Schinke, Photodissociation Dynamics (Cambridge University Press, Cambridge, 1993).

${ }^{18}$ J. Janszky and An. V. Vinogradov, Phys. Rev. Lett. 64, 2771 (1990).

${ }^{19}$ I. Averbukh and M. Shapiro, Phys. Rev. A 47, 5086 (1993).

${ }^{20}$ M. Dantus, M. J. Rosker, and A. H. Zewail, J. Chem. Phys. 87, 2395 (1987).

${ }^{21}$ M. J. Rosker, M. Dantus, and A. H. Zewail, Science 241, 1200 (1988).

${ }^{22}$ S. O. Williams and D. G. Imre, J. Phys. Chem. 92, 6648 (1988).

${ }^{23}$ R. Heather and H. Metiu, Chem. Phys. Lett. 157, 505 (1989).

${ }^{24}$ M. D. Feit, J. A. Fleck, and A. Steiger, J. Comput. Phys. 47, 412 (1982).

${ }^{25}$ V. Engel, Comput. Phys. Commun. 63, 228 (1991). 